\title{
Corynebacterium Urealyticum Bacteremia in A Hemodialysis Patient with Perm Catheter Infection
}

\author{
Kai Yao Yang ${ }^{1}$, Yen Cheng Yeh ${ }^{1}$, Chai Chao $\mathrm{Wu}^{2}$, Chih Chiang Wang ${ }^{1,2}$ and Chen Yi Liao*1,2 \\ ${ }^{1}$ Department of Medicine, Kaohsiung Armed Forces General Hospital, Taiwan \\ ${ }^{2}$ Division of Nephrology, Department of Internal Medicine, Tri-Service General Hospital, National Defense Medical Center, Taiwan
}

Received: 制: April 07, 2018; Published: 制 September 12, 2018

*Corresponding author: Chen Yi Liao, Division of Nephrology, Department of Internal Medicine, Kaohsiung Armed Forces General Hospital, No. 2, Zhongzheng 1st Road, Lingya Dist, Kaohsiung City 802, Taipei, Taiwan

Abstract

Aim: The aim of this stud was to present a case with unusual Corynebacterium infection with recurrent perm catheter infection

Back Ground: Corynebacterium urealyticum (Corynebacterium CDC group D2 bacterium) is an aerobic, catalase-positive, gram positive bacillus with the typical appearance of diphtheroids that is unable to acidify carbohydrate, has strong urease activity, and is resistant to multiple antibiotics. It has been associated mainly with infections of the urinary tract. The isolation of this organism in cases of other infection is highly unusual.

Case Description: A 72-year-old female perm catheter infection due to a case of C. urealyticum that had developed recurrent spiking fever while undergoing hemodialysis through the subclavian catheter site. The final wound and blood cultures yielded C. urealyticum, which was confirmed to be a catheter-related bloodstream infection.

Conclusion: Appropriate antibiotic therapy and immediately removal of perm catheter is of paramount importance in C. urealyticum catheter infection. Clinical significance: persistent perm site redness and unknown origin infection should always put in perm catheter infection in mind.

Keywords: Corynebacterium urealyticum; Perm catheter infection

\section{Background}

Corynebacteria are gram positive, catalase positive, non-spore forming, non-motile bacilli that are frequently considered as contamination when they grow in blood culture, as they are found in the mucosa and on the skin of humans. The genus contains the species Corynebacterium diphtheria and the nondiphtherial corynebacteria, collectively referred to as diphtheroids. Non-diphtherial corynebacteria, originally thought to be mainly contaminants, but with growing numbers of immunocompromised hosts, are now recognized as a new pathogen as formerly presumed [1]. The infamous non-diphtherial corynebacteria can cause diseases including Corynebacterium ulcerans, Corynebacterium jeikeium (group JK), Corynebacterium urealyticum (D2), Corynebacterium pseudotuberculosis, Corynebacterium hemolyticum, Corynebacterium striatum and Corynebacterium pseudodiphtheriticum. Both C. urealyticum and C. jeikeium are strict aerobes that exhibit lipid-requiring and multidrug-resistance phenotypes. However, C. jeikeium can be differentiated from C. urealyticum because it is non-ureolytic and produces acid from glucose [2]. Corynebacterium jeikeium and Corynebacterium urealyticum are well-established human pathogens exhibiting resistance to several antibiotics.
Although formerly considered as a contaminant, C. urealyticum is being increasingly reported as the causative organism of many infections, especially in those at risk [3]. Corynebacterium urealyticum (CU) (formally the Corynebacterium group D2) is a strong urease activity that can infect the lower (acute or chronic cystitis) and upper (pyelonephritis and encrusted pyelitis) urinary tract [4]. CU is a commensal skin organism that an estimated $12 \%$ of healthy individuals carry, and it has been isolated in $30 \%$ of hospitalized patients [5]. CU converts urea into ammonia, creating alkaline urine, which precipitates struvite and calcium phosphate crystals, forming stones and encrustations on the infected mucosa [5]. They have been reported as causative agents of infective endocarditis with very high mortality rates [6]. Cases have also been described in hemodialysis patients with catheters and graft, with high morbidity and mortality $[7,8]$. Herein, we report one case of CU-related perm catheter infection with successful removal and discharge after empiric antibiotic treatment.

\section{Case Description}

A 72-year-old female with history of hypertension, type 2 diabetes mellitus, coronary artery disease, end-stage renal disease 
status post hemodialysis via right subclavian perm catheter for 2 years, hyperthyroidism status post operation and adenocarcinoma of hepatic flexure colon status post right hemicolectomy presented to the emergency department with a one-day history of fever during hemodialysis. She also experienced poor appetite, fatigue and tenderness on the perm catheter insertion site. Vital signs revealed high body temperature $\left(38.2^{\circ} \mathrm{C}\right)$, rapid heart rate and normal blood pressure. Physical examination showed an erythematous skin change on right subclavian perm catheter insertion site Laboratory investigation revealed leukocytosis of $12.6 \mathrm{x} 103 / \mu \mathrm{L}$ (normal 4.0 to 11.0 ) with marked elevation of neutrophils (90.6\%), total bilirubin $0.65 \mathrm{mg} / \mathrm{dL}$ (normal 0.3 to 1.0), alanine aminotransferase (ALT) $18 \mathrm{U} / \mathrm{L}$ (normal 7 to 52), aspartate aminotransferase (AST) $14 \mathrm{U} / \mathrm{L}$ (normal 13 to 39 ), creatinine $1.6 \mathrm{mg} / \mathrm{dL}$ (normal 0.7 to 1.3),C-reactive protein

$6.81 \mathrm{mg} / \mathrm{dL}$ (normal <1.0), and albumin $2.7 \mathrm{~g} / \mathrm{dL}$ (normal 3.5 to 5.7$)$.

Urine examination showed proteinuria (3+), leukocyturia (urine WBC of 50-99/HPF) and mild erythrocyturia (RBC of 2-5/ HPF). An initial chest radiograph showed pattern of pulmonary congestion. The patient was presumptively diagnosed with urinary tract infection and was administrated with ceftazidime $1.0 \mathrm{~g}$ intravenous route once every day and vancomycin $1.0 \mathrm{~g}$ intravenous route once every 5 days. The patient had experienced recurrent spiking fever especially when undergoing hemodialysis via perm catheter. Due to progressive erythematous change of skin around the area of perm catheter implantation and painful sensation told by the patient, perm catheter removed 5 days after admission due to highly suspected catheter-related bloodstream infection. She had Enterobacter aerogenes related urinary tract infection in the past but routine urine cultures were sterile at this admission.

The final blood and wound cultures from the removed perm catheter yielded CU. Antimicrobial susceptibility tests were performed by the Kirby-Bauer disk dilution method on MuellerHinton agar with $5 \%$ sheep blood. The organism was susceptible to ciprofloxacin, clindamycin, erythromycin, oxacillin, penicillin G, rifampicin, tetracycline, tigecycline and vancomycin. Investigation for mycobacteria, fungi and viruses was unremarkable. Echocardiography revealed no evidence of valve vegetation but severe mitral regurgitation and moderate tricuspid regurgitation. Due to the confirmed pathogen, we maintained intravenous vancomycin treatment for 4 weeks and another new perm catheter was implanted on the left subclavian vein after 16 days of admission. The patient was successfully discharged without any sequelae.

\section{Discussion}

CU is known to be a natural colonizer of the human skin and urinary tract, especially in patients receiving broad-spectrum antibiotics [3]. It is usually missed in routine culture because of its diphtheroid morphology (so considered a contaminant) and it requires 48 hours of incubation to grow [2]. CU is currently a common pathogen usually associated with infections of the urinary tract to cause acute cystitis, pyelonephritis, alkaline encrusted cystitis, and encrusted pyelitis $[9,10]$. Reports of non-urinary infections caused by this organism are highly unusual [2] such as bacteremia [11], endocarditis, pericarditis [12], postsurgical osteomyelitis, peritonitis in outpatients with chronic peritoneal dialysis, wound infections $[11,12]$ and it is even observed in kidney transplant groups [13]. Corynebacterium species has recently been shown to be associated with peritoneal catheter infection [14], and perm catheter infection during hemodialysis [7,8] After conducting a review of the English literature from Pubmed, we found that the connection between $\mathrm{CU}$ and hemodialysis has never been mentioned before. Bacteremia due to $\mathrm{CU}$ has been reported with a mortality rate up to $20 \%$ [14].

According to Pagnoux et al., risk factors of CU infection are summarized as prolonged use of a urinary catheter, hospitalization for long periods, immunocompromised patients, kidney transplantation, recent urologic procedure, chronic debilitating disease, broad spectrum antibiotic treatment, cytotoxic drug usage and history of previous urinary tract infection [3]. Our patient was a chronic hemodialysis case with frequent hospitalization and one episode of urinary tract infection caused by Enterobacter aerogenes but without recent Foley catheter use. Tracing back the patient's past history, she indeed received wide spectrum antibiotic treatment due to episode of infection including urinary tract infection, pneumonia and perm catheter infection.

Type 2 diabetes mellitus and colon adenocarcinoma in our patient contributed to her immunocompromised states. Positive blood culture with Corynebacterium species should prompt an evaluation for endocarditis, starting with a transthoracic echocardiogram, and if the suspicion is still high, a transesophageal echocardiogram should be done. This is because of high mortality associated with endocarditis caused by this organism. According to a study by Belmares et al., up to $28 \%$ of patients required valve replacement and $43.5 \%$ died [15]. Thus, if CU endocarditis is confirmed, valve replacement in conjunction with a 4 -week duration of empirical antibiotic use is mandatory. The majority of CU strains are highly resistant to a large number of antibiotics, including aminoglycosides, B-lactams, and macrolides and variably susceptible to fluoroquinolones, macrolides, ketolides, rifampin, and tetracyclines [16]. CU strains are, however, uniformly susceptible to the glycopeptides like vancomycin and teicoplanin. Glycopeptide alternatives, linezolid and quinupristin-dalfopristin are also proven to be effective in vitro against multi-drug resistant CU strains [16].

According to the study by Garcia-Bravo et al, the percentage of resistance to most of the tested antibiotics was significantly higher in the strains of $\mathrm{CU}$ isolated from hospitalized patients than in those from non-hospitalized patients. These factors are unknown, but hospitalization and previous use of antibiotics could play a role in the appearance of resistance in this microorganism $[4,16]$. The perm catheter culture and blood culture in our patient showed $\mathrm{CU}$, which confirmed the diagnosis of perm catheter-related bloodstream infection caused by CU. In patients with catheterrelated bacteremia contributed by $\mathrm{CU}$, removal of the catheter in conjunction with empiric antibiotic administration such as vancomycin are considered as major treatment regimes [11]. Our 
patient also successfully recovered after courses of vancomycin in conjunction with catheter removal and implantation of another perm catheter for temporary hemodialysis.

\section{Conclusion}

In conclusion, isolated presence of Corynebacterium species in hemodialysis patients should not be written off as contaminants. Subtle symptoms like loss of appetite, weight loss and fever especially occurring during hemodialysis should prompt a thorough evaluation, including an echocardiogram before uncommon organisms are isolated. When catheter infection caused by CU is confirmed, the infective perm catheter should be removed as well as empiric antibiotics active against CU, mainly vancomycin, should be administered.

\section{References}

1. Riegel P, de Briel D, Prévost G, Jehl F, Monteil H (1994) Genomic diversity among Corynebacterium jeikeium strains and comparison with biochemical characteristics and antimicrobial susceptibilities. J Clin Microbiol 32(8): 1860-1865.

2. Salem N, Salem L, Saber S, Ismail G, Bluth MH (2015) Corynebacterium urealyticum: a comprehensive review of an understated organism. Infect Drug Resist 21(8): 129-145.

3. Pagnoux C, Bérezné A, Damade R, Paillot J, Aouizerate J, et al. (2011) Encrusting cystitis due to Corynebacterium urealyticum in a patient with ANCA-associated vasculitis: case report and review of the literature. Semin Arthritis Rheum 41(2): 297-300.

4. Garcia-Bravo M, Aguado JM, Morales JM, Noriega AR (1996) Influence of external factors in resistance of Corynebacterium urealyticum to antimicrobial agents. Antimicrob Agents Chemother 40(2): 497-499.

5. Chung SY, Davies BJ, O’Donnell WF (2003) Mortality from grossly encrusted bilateral pyelitis, ureteritis, and cystitis by Corynebacterium group D2. Urology 61(2): 463.

6. Belmares J, Detterline S, Pak JB, Parada JP (2007) Corynebacterium endocarditis species-specific risk factors and outcomes. BMC Infect Dis $7(4)$.

ISSN: 2574-1241

DOI: 10.26717/BJSTR.2018.09.001735

Chen Yi Liao. Biomed J Sci \& Tech Res

(C) (i) This work is licensed under Creative

Submission Link: https://biomedres.us/submit-manuscript.php
7. Martinez Vea A, Costa J, Garcia C, Bardají A, Ridao C, et al. (1993) Corynebacterium group JK endocarditis in a haemodialysis patient. Nephrol Dial Transplant 8(2): 177-179.

8. Ifantidou AM, Diamantidis MD, Tseliki G, Angelou AS, Christidou P, et al. (2010) Corynebacterium jeikeium bacteremia in a hemodialyzed patient. Int J Infect Dis 14(3): 265-268.

9. Aguado JM, Morales JM, Salto E, Lumbreras C, Lizasoain M, et al. (1993) Encrusted pyelitis and cystitis by Corynebacterium urealyticum (CDC group D2): a new and threatening complication following renal transplant. Transplantation 56(3): 617-622.

10. Del Prete D, Polverino B, Ceol M, Vianello D, Mezzabotta F, et al. (2008) Encrusted cystitis by Corynebacterium urealyticum: a case report with novel insights into bladder lesions. Nephrol Dial Transplant 23(8): 2685-2687.

11. Soriano F, Ponte C, Ruiz P, Zapardiel J (1993) Non-urinary tract infections caused by multiply antibiotic-resistant Corynebacterium urealyticum. Clin Infect Dis 17(5): 890-891.

12. Ojeda Vargas M, Gonzalez Fernandez MA, Romero D, Cedrés A, Monzón Moreno C (2000) Pericarditis caused by Corynebacterium urealyticum. Clin Microbiol Infect 6(10): 560-561.

13. López Medrano F, García Bravo M, Morales JM, Andrés A, San Juan R, et al. (2008) Urinary tract infection due to Corynebacterium urealyticum in kidney transplant recipients: an underdiagnosed etiology for obstructive uropathy and graft dysfunction-results of a prospective cohort study. Clin Infect Dis 15,46(6): 825-830.

14. Núñez Moral M, Sánchez Álvarez E, González Díaz I, Peláez Requejo B, Fernández Viña A, et al. (2014) Exit-site infection of peritoneal catheter is reduced by the use of polyhexanide. results of a prospective randomized trial. Perit Dial Int 34(3): 271-277.

15. Belmares J, Detterline S, Pak JB, Parada JP (2007) Corynebacterium endocarditis species-specific risk factors and outcomes. BMC Infect Dis 6(7): 4.

16. Tauch A, Trost E, Tilker A, Ludewig U, Schneiker S, et al. (2008) The lifestyle of Corynebacterium urealyticum derived from its complete genome sequence established by pyrosequencing. J Biotechnol 31;136(1-2): 11-21.

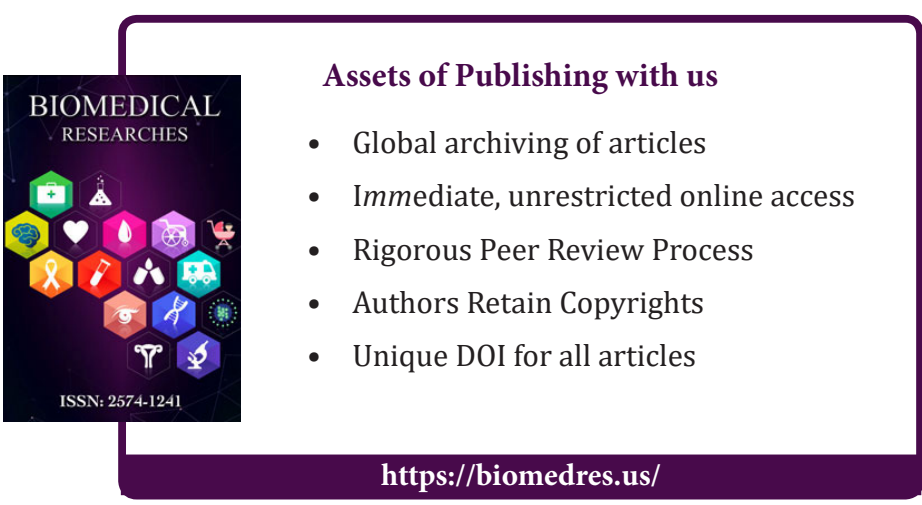

